\title{
MULTIPASS STEERING PROTOCOLS AT JEFFERSON LAB*
}

\author{
R.M. Bodenstein ${ }^{\#}$, M.G. Tiefenback, JLAB, Newport News, VA 24606, U.S.A.
}

\section{Abstract}

The CEBAF recirculating accelerator consists of two CW superconducting RF linacs, through which an electron beam is accelerated for up to 5 passes. Focusing and steering elements affect each pass differently, requiring a multipass steering protocol to correct the orbits. Perturbations include lens misalignments (including long-term ground motion), BPM offsets, and focusing and steering from RF fields inside the cavities. A previous treatment of this problem assumed all perturbations were localized at the quadrupoles and the absence of $x-y$ coupling. Having analyzed the problem and characterized the solutions, we developed an empirical iterative protocol to compare against previous results in the presence of skew fields and cross-plane coupling. We plan to characterize static and accelerationdependent components of the beam line perturbations to allow systematic and rapid configuration of the accelerator at different linac energy gains.

\section{CEBAF}

In much of the CEBAF (Continuous Electron Beam Accelerator Facility) accelerator, beams of different energies pass through independent transport lines. However, the linacs themselves house all five energies in one beam pipe, in which all five beams are ideally coincident. However, magnetic perturbations from the Earth's field and focusing magnet misalignment, dipole fields in accelerating cavities, and other sources cause energy dependent angular deflections. The cumulative effect of these perturbations can vary with beam energy because of differences in the wavelength of the betatron orbit oscillations. A simple algorithm has been devised which adjusts the sparse set of available correctors in a way which is asymptotically correct at high energy. This algorithm has been successfully tested manually, and will be automated for use in the accelerator to minimize systematic orbit errors in the dipoles which separate and recombine beams of different energy.

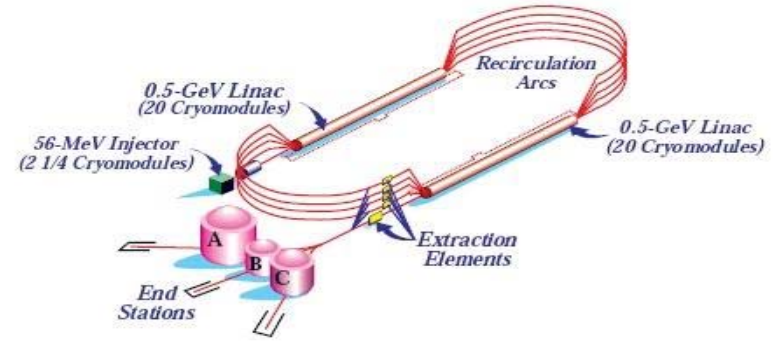

Figure 1

\footnotetext{
* Authored by Jefferson Science Associates, LLC under U.S. DOE Contract No. DE-AC05-06OR23177. The U.S. Government retains a non-exclusive, paid-up, irrevocable, world-wide license to publish or reproduce this manuscript for U.S. Government purposes.

"ryanb@jlab.org
}

\section{PRESENT APPROACH}

The default protocol for positioning the beams in the linacs is to steer the first pass beam close to the axis of each linac, then adjust the injection conditions for each higher pass beam to minimize the overall deviation of its trajectory from the axis. This approach presumes that the beam line is straight. However, magnet positioning errors and ground motion over time perturb the orbit from the ideal. This method has resulted in deviation of the beam position at injection and exit by several millimeters from the ideal axis. The $6 \mathrm{GeV}$ system is able to tolerate these systematic offsets at the injection and exit points of the linacs, as will the lowest energy beams in the $12 \mathrm{GeV}$ system. However, for the higher energy parts of the 12 $\mathrm{GeV}$ system, the steering tolerance decreases to only 1 $\mathrm{mm}$, partly due to the larger anticipated beam size (from synchrotron radiation heating) and partly from the need to avoid exposing the beam to multipole fringe fields to avoid halo generation. A long-term, procedural solution to configuring the accelerator common beam lines must be provided to satisfy the needs of the accelerator for the $12 \mathrm{GeV}$ upgrade.

\section{DEVELOPMENT OF PROTOCOL}

Previous treatments were not user friendly and the supporting software had not been maintained across changes in the underlying software base. A simpler and more intuitive protocol was pursued, amenable to both manual implementation and an automated approach. It is expected to provide baseline data which can be used for model-based configuration at different energies.

A spreadsheet was created to simulate the beam positions at each BPM, including BPM and quadrupole alignment errors, as well as other unknown perturbations. As may be seen from first principles, the only way to make the trajectories of beams at different energy coincident is for both to follow the magnetic centers of the linac, and for these magnetic centers to lie along a straight line. Using available correctors to make any two beams of different energy coincident at defined injection and exit points defines the ideal line, along which the other passes may be directed by injection steering. If the steering perturbations existed only local to the corrector magnets, the correctors so used would displace the effective magnetic centers of the adjacent quadrupoles to lie along this straight line. Therefore, the only parameters that would be varied are the injected beam position and angle and the strength of the corrector magnets.

In the CEBAF linacs, sequential lenses have alternating horizontal and vertical correctors. The perturbations are distributed along the beam line and cannot be perfectly compensated by sparse correctors. However, the cumulative effect of distributed perturbations is 
progressively well compensated as the beam energy increases and the betatron phase advance decreases. The higher pass beams have long betatron wavelengths, so the effect of distributed steering perturbations along the linac can be compensated with the sparse correctors. This is not so for the lowest energy beam, for which the detailed distribution of these perturbations is important. However, the beam size grows for the higher energy beams in the CEBAF $12 \mathrm{GeV}$ upgrade, and it is these for which orbit control is most critical. If necessary, additional correctors can be installed to smooth the first pass orbit.

An iterative method appeared to be the simplest way to test these ideas. Starting at the entrance of the linac, each corrector was adjusted to align the second and fifth pass beams at the next BPM. If by the end of the linac, the beams did not exit in the desired position, the fifth pass injection position and angle would be adjusted accordingly, and the process would be repeated. Automated correction of the linac in larger segments, possibly each linac as a whole, will be possible with a sufficiently accurate optics model.

\section{EXPERIMENT}

The spreadsheet model did not address all of the limitations of the CEBAF machine, one of which was the available strength of the corrector magnets. This limitation became apparent during the initial test. Two corrector magnets had been reinstalled after maintenance, but offset by $1 / 2 \mathrm{~mm}$ for one, and $1 \mathrm{~mm}$ for the second. When our protocol brought us to these girders, the available strength of the corrector magnets was insufficient to align the beams. After the survey team discovered and corrected this problem, we were able to successfully align the beams. This experience demonstrated that the corrector patterns obtained from this protocol are useful in identifying errors in the line. Once this problem was addressed, the beams were successfully steered to an accuracy of 0.5 millimeters.

\section{DISCUSSION OF RESULTS}

Looking at Figure 2, one can see the positions of the beams prior to the adjustment of the correctors. In Figure $4 \mathrm{a}$, it can be seen that the first pass beam has a very short wavelength (roughly 60 meters), while the wavelength of the second pass beam is longer than the linac. The higher pass beams have even longer wavelengths. This is why the first pass is so greatly influenced by the corrector magnets, while the higher passes continue along their paths with little change.

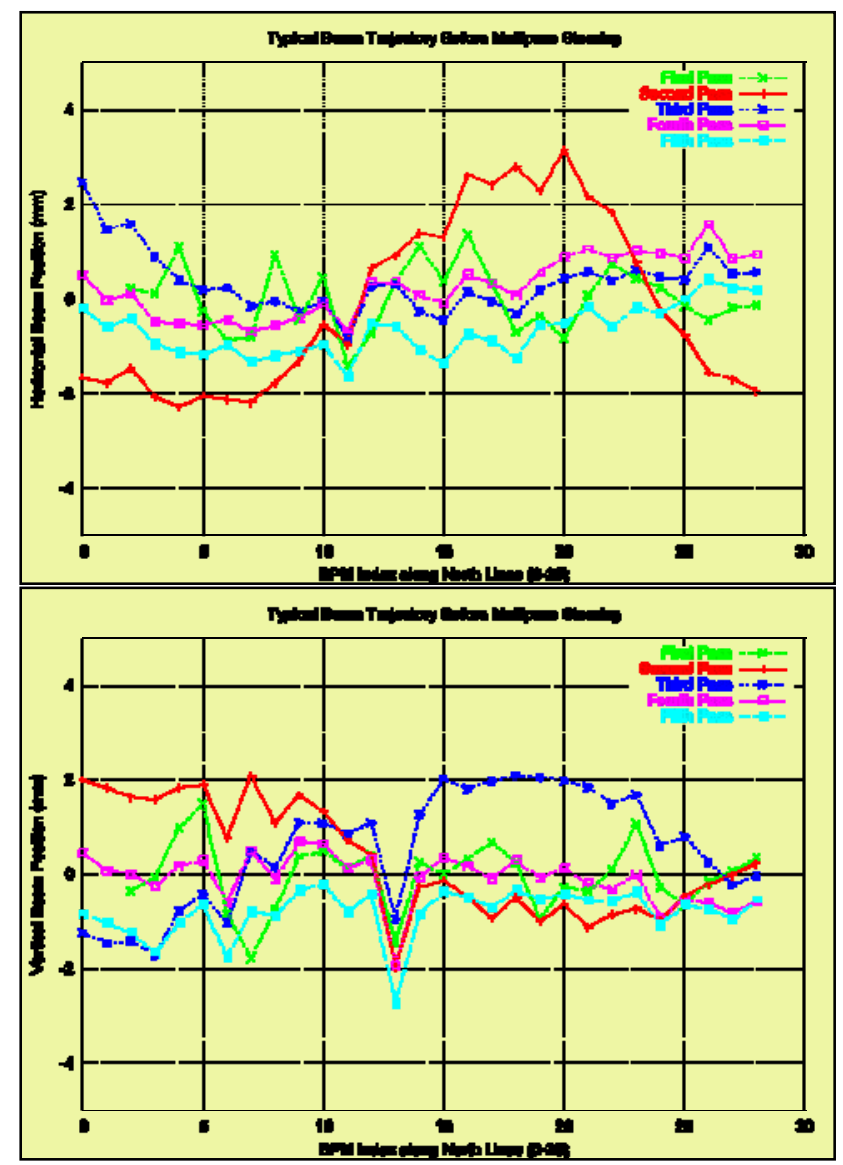

Figures 2a and 2b: Show the $\mathrm{x}$ and $\mathrm{y}$ (respectively) positions of the 5 passes in the beam pipe prior to adjusting the correctors and initial conditions. These are "as found" conditions including the effects of long-term drifts.

With the present deliberately coarse corrector distribution, each corrector compensates for two modules (10 meters each) at a time. The protocol assumes that the perturbations are sufficiently coincident with the correctors. This is sufficiently accurate for the higher passes because of their very large betatron wavelengths, but is not a good approximation for the first pass beam, as demonstrated in Figure 3 . The perturbations are distributed through the acceleration cavities as well as at the lenses, and correcting the cumulative impulse does not work well for the short wavelength first pass beam. This explains the failure of the first pass to coincide with the higher passes. 


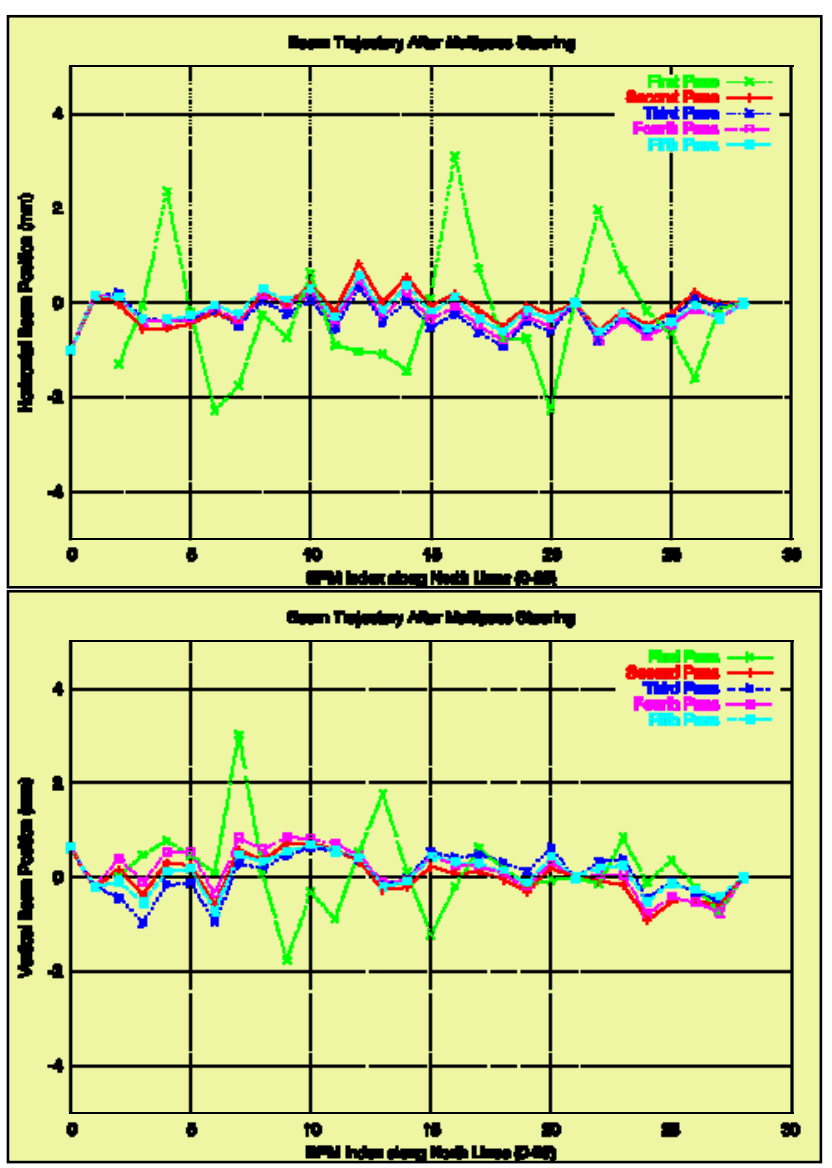

Figures 3a and 3b: Show the $\mathrm{x}$ and $\mathrm{y}$ positions of the 5 passes after manual execution of the protocol. The 4 higher passes are nearly coincident (within $1 / 2 \mathrm{~mm}$ ) throughout the beam pipe. The apparent variations in the positions of the higher passes are the result of systematic offset errors in the BPMs. The beams of different energy cannot all be deflected the same amount by the magnets.

For the $12 \mathrm{GeV}$ upgrade, the high pass beams are larger because of synchrotron radiation heating, and are most critical to position correctly. The first pass beam is smaller and has looser constraints on position control. An earlier algorithm for multipass steering generated a global solution with improved first pass orbit control at the expense of the higher passes. Orbit control of the first pass beam would be improved for the algorithm we propose by using correctors at each quadrupole, but the high pass beams would not benefit.

\section{FUTURE PLANS}

The next step in this process is to correct larger segments of the linac, rather than adjusting one corrector at a time, with the goal being global correction for the whole linac. Individual correctors were initially used to test the concept without requiring large-scale model accuracy. The existing linac optics model does not take into account coupling and skew fields in the cavities, which may be important for global solutions.

If perturbations can be separated into contributions which are static (earth's field), beam energy dependent (such as quadrupole misalignment), and acceleration dependent (cavity dipole fields, etc), then the corrector settings may be "dead-reckoned" for different accelerating gradient distributions along the linac, and for different linac energy gains. Ideally, this will be incorporated into the linac energy management system (LEM), which is responsible for setting the focusing quadrupoles in the linacs, and could be used to set the correctors for best error compensation. A user-friendly tool for use in the control room must be developed. This must be made in a manner that is simple to maintain and use, so that these data may be maintained and extended.

\section{CONCLUSIONS}

It has been verified that the protocol works as expected, and that difficulties in execution may indicate hardware problems in the linac. This protocol provides a global, beam-based reference for BPM zeroes. This protocol can be used to define and monitor a beam-based straight line. This straight line and measured BPM offsets with respect to the local lenses can be used to monitor BPM drift and ground motion over time.

\section{REFERENCES}

[1] Y. Chao, S.A. Bogacz, V.A. Lebedev, "Simultaneous Multiple Pass Steering at Jefferson Lab,” PAC’99, New York, March 1999, p. 738. 